\title{
Conducting clinical studies targeting cognition in psychiatry: guiding principles and design
}

\author{
Tamsyn E. Van Rheenen, ${ }^{1,2,3}$ Kathryn E. Lewandowski, ${ }^{4,5}$ Jessica M. Lipschitz, ${ }^{5,6}$ \\ and Katherine E. Burdick ${ }^{5,6 *}$
}

\footnotetext{
${ }^{1}$ Department of Psychiatry, Melbourne Neuropsychiatry Centre, University of Melbourne, Melbourne, Australia

${ }^{2}$ Centre for Mental Health, Faculty of Health, Arts and Design, School of Health Sciences, Swinburne University, Melbourne, Australia

${ }^{3}$ Cognitive Neuropsychiatry Laboratory, Monash Alfred Psychiatry Research Centre (MAPrc), The Alfred Hospital and Central Clinical School,

Monash University, Melbourne, Australia

${ }^{4}$ Schizophrenia and Bipolar Disorder Program, McLean Hospital, Belmont, Massachusetts, USA

${ }^{5}$ Department of Psychiatry, Harvard Medical School, Boston, Massachusetts, USA

${ }^{6}$ Department of Psychiatry, Brigham and Women's Hospital, Boston, Massachusetts, USA
}

Cognitive dysfunction is common in many psychiatric disorders. While it has long been described as a core feature in schizophrenia, more recent data suggest qualitatively similar impairments in patients with bipolar disorder and major depressive disorder. There is compelling evidence to suggest that cognitive impairment contributes directly to functional disability and reduced quality of like across these disorders. As current treatments focus heavily on "primary" symptoms of mood and psychosis, the standard of care typically leaves cognitive deficits unmanaged. With this in mind, the field has recently begun to consider intervening directly on this important symptom domain, with several ongoing trials in schizophrenia. Fewer studies have targeted cognition in bipolar disorder and still fewer in MDD. With progress toward considering this domain as a target for treatment comes the need for consensus guidelines and methodological recommendations on cognitive trial design. In this manuscript, we first summarize the work conducted to date in this area for schizophrenia and for bipolar disorder. We then begin to address these same issues in MDD and emphasize the need for additional work in this area.

Received 8 February 2018; Accepted 26 February 2018; First published online 24 September 2018

Key words: Bipolar disorder, clinical trial, cognition, depression, schizophrenia, treatment.

Cognitive impairment is common in several neuropsychiatric disorders to a varying degree, including schizophrenia (Sz), bipolar disorder (BD), and major depressive disorder (MDD). Deficits in core cognitive domains have been consistently shown to strongly predict disability in occupational, social, and community functioning. While some symptoms of major psychiatric disorders are relatively well-controlled by existing treatments, cognitive impairment has been largely unresponsive to standard interventions. The time is ripe for studies that directly target this important domain to promote full recovery.

\footnotetext{
* Address for correspondence: Katherine E. Burdick, PhD, Brigham and Women's Hospital, 221 Longwood Ave., Boston, MA 02119, USA. (Email: kburdick1@bwh.harvard.edu)
}

\section{Cognitive Trial Design in Sz and BD}

Over recent years there has been increasing research interest directed toward the treatment of cognitive impairment in $\mathrm{Sz}^{1-3}$ and BD. ${ }^{4,5}$ While long considered a hallmark symptom of $\mathrm{Sz}$, cognitive deficits have only more recently become recognized as a core feature of BD. ${ }^{6,7}$ Characteristically, cognitive impairment in $\mathrm{Sz}$ is known to be diffuse and pervasive, affecting multiple domains and representing a profound negative deviation from normative samples in the order of between 1 and 2 standard deviations or more. ${ }^{8}$ Current evidence suggests qualitative similarities in this profile compared with BD, although the magnitude of effects in BD is typically smaller (though not always). ${ }^{9-12}$ It is well known that cognitive deficits confer a substantial contribution to functioning over and above clinical symptoms in both disorders, and although there is a clear need for novel drug development and novel psychological 
interventions, currently there remain no pharmacological agents with indications specifically for this symptom dimension.

The MATRICS initiative was introduced over a decade ago to promote the development of pharmacological agents that target neurocognitive impairment in $\mathrm{Sz}$ as an independent domain. This was a collaborative effort between the National Institute for Mental health (NIMH), academia, and regulatory agencies (US Food and Drug Administration - FDA) in an effort to establish guidelines for drug development for a newly recognized indication-cognitive impairment associated with schizophrenia (CIAS).

Initial guidelines proposed at the 2005 FDA-NIMHMATRICS workshop on clinical trial design for neurocognitive drugs for $\mathrm{Sz},{ }^{2}$ and then updated 5 years later, ${ }^{1}$ focused on patient characteristics, treatment agent choice, screening assessments, primary outcome measures, and trial-specific characteristics, where recommendations included the following:

1. Inclusion of medication-stable $\mathrm{Sz}$ patients in nonacute stages of illness, with no more than moderate formal thought disorder and moderate-severe positive symptoms, and minimal extrapyramidal and depressive symptoms. This was considered important to reduce the likelihood of clinical change during treatment that could confound trial results.

2. Basing the choice of adjunctive antipsychotic medication on pharmacodynamic and pharmacokinetic interactions with the treatment agent dependent on trial stage — with interacting agents avoided at stage I and interactions examined at stage II in an "all-comers" design.

3. Allowing for concomitant antipsychotic use (ie, polypharmacy) provided pharmacokinetic or pharmacodynamics effects are considered.

4. Exclusion of patients with illicit drugs use in early stage trials, and excluding patients whose level of impairment is limited or so extreme (based on a screening measure different from the primary trial outcome measure) that it impacts outcome validity owing to ceiling effects or an inability to receive benefit from the intervention.

5. The use of the MATRICS Consensus Cognitive Battery (MCCB)—specifically designed to assess cognitive change in $\mathrm{Sz}$ - was recommended as the primary cognitive measure, supplemented by inclusion of a co-primary measure of functioning or subjective cognitive performance characterized by good face validity, reliability, tolerability, association with cognition and community functioning and with expected sensitivity to change in a timeframe proximal to that of the primary cognitive measure. Given the "distance" of functional measures from the biological impact of cognition enhancing drugs, clear separation of impact by the treatment agent on the co-primary measure was considered especially important in the context of decision rules that quantify trial outcomes as either positive or negative.

6. By this same token, it was recommended that pilot data and psychometric attributes should inform the pre-specification of either a global or domain-specific primary cognitive outcome measure, with more rather than fewer testing sessions included in the design to reduce attrition.

7. Finally, placebo or cognitively neutral comparators were recommended for adjunctive and broadspectrum agents respectively, with the trial needing to last long enough to confer enduring effects on cognition of 6 months or longer.

In the context of these recommendations and subsequent advances in the creation of a standardized cognitive test battery for assessing cognitive change in Sz, the International Society of Bipolar Disorders (ISBD) convened a taskforce to identify a preliminary battery of tests for use in research on BD. ${ }^{13}$ From this it was decided that the MCCB could be usefully applied to the disorder, with the addition of more complex measures of verbal learning or executive function as a complement. In combination, these measures form a battery known as the ISBD-battery for the assessment of neurocognition (ISBD-BANC). ${ }^{14}$ The MCCB tests were subsequently validated in $\mathrm{BD},{ }^{15,16}$ and following this, a smaller consensus panel of experts proposed preliminary guidelines for cognitive trial design, drawing on previous efforts in $\mathrm{Sz}$ but focusing on the unique nuances of BD. ${ }^{6}$ These guidelines, which address diagnostic (subtypes and comorbidities), clinical (symptoms, medications), and measurement issues (cognitive and functional outcome measures), were recently expanded in the International Society of Bipolar Disorders Targeting Cognition Taskforce methodological recommendations paper and associated commentaries. ${ }^{14,17,18}$

Notable suggestions from these papers involve specific guidance regarding the use of certain classes of pharmacological agents for clinical trial design, and the following other recommendations:

1. Inclusion of a control group, and trial enrichment of cognitively impaired patients that deviate by at least half a standard deviation below normative means

2. Selection of euthymic or affectively stable participants on a stable treatment regime, keeping lithium within therapeutic range and allowing concomitant medications while limiting high doses of antipsychotic, anti-dopaminergic, and anti-cholinergic agents with cognitive side-effects

3. Inclusion of a composite score of tests from or equivalent to the ISBD-BANC as the primary outcome, from which small-medium effects can be considered clinically important 
4. Including a functional assessment as a secondary measure, provided it has clear relevance to cognitive change expected on account of the specific intervention, and including a social cognitive outcome to assess broader cognitive change indirectly resulting from the intervention

5. Administering biological interventions for 6-12 weeks and psychological interventions for 10-21 weeks, with follow-up 3-6 months following to assess functional change that may have a time-lag

6. Incorporating symptom change as a covariate in statistical analyses to address pseudospecificity, particularly for biological interventions where this issue has a greater chance of confounding results.

The existing methodological recommendations for cognitive trial design in both $\mathrm{Sz}$ and $\mathrm{BD}$ draw on common threads, including the need to recruit clinically stable patients, trial enrichment with patients evidencing meaningful cognitive deficits, the use of a standardized outcome battery for uniform assessment across samples and trials, and the need for secondary or co-primary measures of functioning. Guidance around psychological treatment trial design is still lacking in BD however, and thoughts around this are needed to advance the field.

\section{Cognitive Dysfunction in MDD}

As is the case with $\mathrm{Sz}$ and BD, cognitive impairment has been increasingly recognized as a core feature of major depressive disorder (MDD), with serious impacts on psychosocial functioning and clinical course. ${ }^{19}$ The Sequenced Treatment Alternatives to Relieve Depression $\left(\mathrm{STAR}^{*} \mathrm{D}\right)$ study found that nearly $90 \%$ of the 2541 patients enrolled reported cognitive difficulties such as problems with concentration and decision making. ${ }^{20}$ Patients with MDD experience a broad range of cognitive deficits across domains, including verbal processing, attention, learning, memory, and several aspects of executive functioning including set shifting, working memory, and response inhibition. ${ }^{21-25}$ While the magnitude of cognitive impairments differs by cognitive domain, effect sizes compared to healthy controls appear to be in the medium to large range. ${ }^{26}$

Cognitive deficits are present early in the course of illness, likely by illness onset, ${ }^{27,28}$ and often persist during clinical remission. ${ }^{20,29-31}$ While some aspects of cognition may be influenced by mood state (eg, psychomotor speed, memory), others are relatively independent of state mood symptoms (eg, attention, executive functioning). ${ }^{27,30}$

Overall illness severity is associated with cognitive dysfunction in MDD, as has been reported in other affective disorders. Markers of overall illness burden including history of psychosis, ${ }^{29}$ number and total duration of depressive episodes, ${ }^{29,31}$ severity of depressive symptoms, and melancholic subtype ${ }^{30}$ are associated with greater cognitive impairment.

Cognitive deficits are present at similar rates in patients with MDD and other serious mental illnesses such as $\mathrm{Sz}$ and $\mathrm{BD},{ }^{32}$ and are qualitatively similar, with evidence of impaired underlying attention, verbal learning, and executive functioning across diagnoses. ${ }^{19}$ However, the severity of impairment differs quantitatively among these disorders, with patients with MDD showing the least severe deficits. ${ }^{25}$ In terms of longitudinal course, a cluster analytic approach grouping patients with $\mathrm{Sz}, \mathrm{BD}$, or MDD based on cognitive change over follow up found no difference in diagnostic makeup of each cluster, suggesting that cognitive trajectories are not diagnosis-specific. ${ }^{28}$

As has been shown for $\mathrm{Sz}$ and $\mathrm{BD},{ }^{33,34}$ cognitive impairments are strongly associated with psychosocial functioning across multiple domains in patients with MDD. ${ }^{24,35-39}$ Not only do cognitive deficits impede community functioning directly, they may also increase rates of recurrent depressive episodes, leading to an overall worsening course of illness. ${ }^{23,24}$

Together these findings demonstrate that cognitive functioning is broadly impaired in patients with MDD, affecting a large majority of patients. Deficits are present early in the course of illness, persist during remission of mood symptoms, and significantly impact community functioning and illness course. Thus, cognitive dysfunction in MDD represents an important target for improving both psychosocial functioning and overall illness trajectory.

\section{Cognitive Treatment Considerations for MDD}

Many of the same issues arise when considering targeting cognition in MDD as have been previously discussed for SZ and for BD. Key recommendations that would also apply to MDD trials include the following:

1. The need to pre-screen to identify those patients who are most likely to benefit from a cognitive intervention based on the presence of objective impairment at baseline. This may be of greatest utility in MDD where base rates of cognitive impairment are likely lower than those noted in Sz and BD.

2. Inclusion of a functional measure as co-primary to evaluate the relationship between the intervention's effects on cognition and on functional outcome

3. Exclusion of patients with current substance misuse and other comorbidities that might interfere with cognition

4. Exclusion of certain medications with known cognitive effects (either positive or negative) and keeping all other concomitant medication stable. 
Where some of the recommendations might diverge from those made for SZ and BD is also of interest. In general, it is thought that cognitive deficits should be targeted independent of the core symptoms of the illness (eg, in euthymic or remitted patients); however, there is at least one clear example where depression and cognition were evaluated in parallel-a design that still allowed for sufficient evidence to warrant consideration of an indication for cognition (see below for vortioxetine example). While pseudo-specificity still remains a concern if an agent is seeking an independent indication as pro-cognitive, it would be clinically important to identify agents that target both mood and cognition in parallel. Presumably the improvement of both mood and cognitive aspects of a depressive episode will allow for a quicker and more complete functional recovery than would the improvement of mood alone. Another issue that might differentiate cognitive trials in MDD from those in Sz or BD is the use of monotherapy. Most trials in $\mathrm{Sz}$ and in $\mathrm{BD}$ are adjunctive, requiring additional medications (eg, mood stabilizers and/or antipsychotics) to ensure safety and to avoid exacerbation of psychotic and manic symptoms. While there are always risks involved in intervention trials, MDD may be more amenable to monotherapy designs than are $\mathrm{Sz}$ and $\mathrm{BD}$, which would allow for the evaluation of agents without the confounding effects of concomitant medications. This will, of course, depend on the type of agent being tested and its mechanism of action and side effect profile.

To date, there are very few, if any, clinical trials that have included cognition as a primary outcome in patients with MDD; however, there is a growing interest in including cognition as a secondary outcome. One example of how this approach might be mutually beneficial to pharmaceutical companies and to the patients who seek treatment for depression comes from a series of studies of vortioxetine. In 2014, vortioxetine received FDA approval for MDD. Secondary analyses from the pivotal depression studies focused on the cognitive outcome measures that were included as secondary. Results suggested pro-cognitive effects of vortioxetine in patients with MDD, and data were sufficient to warrant approval of a new indication for cognitive impairment in adults with MDD by European agencies. The FDA ultimately rejected the claim; however, in the process of considering the evidence, the FDA formally acknowledged that cognitive impairment in a disorder other than $\mathrm{Sz}$ (MDD in this case) was worthy of a drug target claim. These regulatory changes reflect a changing tide in drug development and emphasize the importance of identifying new drug targets that span a broad range of major psychiatric disorders.

\section{Future Directions}

The recommendations put forth in this article are largely focused on the potential use of pharmacological interventions for cognitive trials. The use of psychosocial approaches and cognitive remediation are also of great interest but outside of the scope of this manuscript. One area of emerging interest that is worth a brief comment here is the growing use of digitalized cognitive training (CT) interventions. While investigation of digitalized CT in MDD is relatively new, it follows in the footsteps of a relatively large literature base supporting the efficacy of digitalized CT interventions in patients with schizophrenia. ${ }^{40}$ A recent meta-analysis of the impact of computerized CT in depressive disorders identified 9 relevant randomized trials and found positive effects. ${ }^{35}$ This meta-analysis assessed the impact of computerized CT on 5 domains of cognitive functioning. Significant moderate to large effect sizes were observed in the areas of attention (Hedge's $\mathrm{g}=0.67$ ), working memory (Hedge's $g=0.72$ ), and global functioning (Hedge's $g=1.05)$. Verbal memory, despite being targeted in half of the studies included in this meta-analysis, did not improve with computerized CT, and neither did executive functioning (although only 1 study was found that targeted this domain, and it was not a major focus of the intervention in that study). The same meta-analysis identified significant effects of computerized CT on depressive symptom severity (Hedges $g=0.43$ ) and daily functioning (Hedge's $\mathrm{g}=0.72$ ).

More recently, studies of CT delivered via a mobile app have demonstrated improvements in cognitive functioning as well as mood symptoms. Compared to CT designed for use on computers, CT delivered via mobile app is more accessible and provides the opportunity for real-time feedback and prompting. Project: $\mathrm{EVO}^{\mathrm{TM}}$, a video-game mobile app designed to enhance cognitive control, improved working memory and attention in individuals with late-life depression in a small proof-of-concept trial. ${ }^{41}$ In addition to achieving cognitive improvements, Project: EVO was found to improve mood and self-reported functioning both in older adults and a general adult population with depression. ${ }^{41,42}$ Other app-based interventions for conditions and constructs related to cognition, such as organization, attentional control, memory, and mindfulness, have also been associated with improvements in mood in samples not specifically recruited for mood disturbance. ${ }^{43,44}$

Taken together this literature suggests both that digitalized CT can enhance cognition in individuals with depression and that, consistent with findings of overlapping functional neuroanatomy, there is some reciprocity between improvements in depression and cognition. Additional research could support stronger conclusions. 
First, more studies on the efficacy of digitalized CT that include active controls and larger sample sizes are necessary. The meta-analysis conducted by Motter et $a l^{35}$ only included one study with more than 25 participants. Second, more support of transferability from findings on in-program tests to improvements on everyday functioning must be evaluated. Finally, teasing out longitudinal relationships between cognitive improvement and mood improvement from digital CT and variables that may impact the direction of this relationship will allow for more precise tailoring of treatment recommendations.

\section{Conclusions}

In response to the clear and convergent evidence that cognitive impairment is among the most disabling of symptoms in major psychiatric illness, several studies are now underway that target cognition directly in patients with $\mathrm{Sz}$ and $\mathrm{BD}$. There have been working groups established and expert consensus guidelines published that make recommendations to optimize study design for these disorders. A more recent focus on cognition in MDD points toward the need for similar guidelines tailored to this patient sample and supports the need for intervention trials that focus on this relatively understudied aspect of MDD. Here we set the stage for future work in this arena.

\section{Disclosures}

The authors have nothing to disclose.

\section{REFERENCES:}

1. Buchanan RW, Keefe RSE, Umbricht D, Green MF, Laughren T, Marder SR. The FDA-NIMH-MATRICS guidelines for clinical trial design of cognitive-enhancing drugs: what do we know 5 years later? Schizophr Bull. 2011;37(6):1209-1217. doi:10.1093/schbul/sbq038

2. Buchanan RW, Davis M, Goff D, et al. A summary of the FDANIMH-MATRICS workshop on clinical trial design for neurocognitive drugs for schizophrenia. Schizophr Bull. 2005;31 (1):5-19. doi:10.1093/schbul/sbi020

3. Nuechterlein KH, Green MF, Kern RS, et al. The MATRICS Consensus Cognitive Battery, part 1: test selection, reliability, and validity. Am J Psychiatry. 2008;165(2):203-213. doi:10.1176/appi. ajp.2007.07010042

4. Dean OM, Gliddon E, Van Rheenen TE, et al. An update on adjunctive treatment options for bipolar disorder. Bipolar Disord. 2018;20(2):87-96. doi:10.1111/bdi.12601

5. Burdick KE, Braga RJ, Goldberg JF, Malhotra AK. Cognitive dysfunction in bipolar disorder: future place of pharmacotherapy. CNS Drugs. 2007;21(12):971-981.

6. Burdick KE, Goldberg JF, Harrow M, Faull RN, Malhotra AK. Neurocognition as a stable endophenotype in bipolar disorder and schizophrenia. J Nerv Ment Dis. 2006;194(4):255-260. doi:10.1097/01.nmd.0000207360.70337.7e
7. Burdick KE, Ketter TA, Goldberg JF, Calabrese JR. Assessing cognitive function in bipolar disorder: challenges and recommendations for clinical trial design. J Clin Psychiatry. 2015;76 (3):e342-e350. doi:10.4088/JCP.14cs09399

8. Kern RS, Nuechterlein KH, Green MF, et al. The MATRICS consensus cognitive battery, part 2: co-norming and standardization. Am J Psychiatry. 2008;165(2):214-220. doi:10.1176/appi.ajp.2007.07010043

9. Van Rheenen TE, Lewandowski KE, Tan EJ, et al. Characterizing cognitive heterogeneity on the schizophrenia-bipolar disorder spectrum. Psychol Med. 2017;47(10):1848-1864. doi:10.1017/ S0033291717000307

10. Van Rheenen TE, Bryce S, Tan EJ, et al. Does cognitive performance map to categorical diagnoses of schizophrenia, schizoaffective disorder and bipolar disorder? A discriminant functions analysis. $J$ Affect Disord. 2016;192:109-115. doi:10.1016/j.jad.2015.12.022

11. Harvey PD, Siever LJ, Huang GD, et al. The genetics of functional disability in schizophrenia and bipolar illness: methods and initial results for VA cooperative study \#572. Am J Med Genet B Neuropsychiatr Genet. 2014;165B(4):381-389. doi:10.1002/ajmg. b.32242

12. Barch DM, Sheffield JM. Cognitive impairments in psychotic disorders: common mechanisms and measurement. World Psychiatry. 2014;13(3):224-232. doi:10.1002/wps.20145

13. Yatham LN, Torres IJ, Malhi GS, et al. The International Society for Bipolar Disorders-Battery for Assessment of Neurocognition (ISBDBANC). Bipolar Disord. 2010;12(4):351-363. doi:10.1111/j.13995618.2010.00830.x

14. Miskowiak KW, Burdick KE, Martinez-Aran A, et al. Methodological recommendations for cognition trials in bipolar disorder by the International Society for Bipolar Disorders Targeting Cognition Task Force. Bipolar Disord. 2017;19(8):614-626. doi:10.1111/ bdi.12534

15. Burdick KE, Goldberg TE, Cornblatt BA, et al. The MATRICS consensus cognitive battery in patients with bipolar I disorder. Neuropsychopharmacology. 2011;36(8):1587-1592. doi:10.1038/ npp.2011.36

16. Van Rheenen TE, Rossell SL. An empirical evaluation of the MATRICS Consensus Cognitive Battery in bipolar disorder. Bipolar Disord. 2014;16(3):318-325. doi:10.1111/bdi.12134

17. Van Rheenen TE. Commentary on "Methodological recommendations for cognition trials in bipolar disorder by the International Society for Bipolar Disorders Targeting Cognition Task Force." Bipolar Disord. 2018;20(1):72-73. doi:10.1111/ bdi. 12577

18. Lewandowski KE. Hastening treatment development in bipolar disorder: evaluation of consensus-based recommendations for cognition trials. Bipolar Disord. 2018;20(1):70-71. doi:10.1111/ bdi. 12578

19. Millan MJ, Agid Y, Brüne M, et al. Cognitive dysfunction in psychiatric disorders: characteristics, causes and the quest for improved therapy. Nat Rev Drug Discov. 2012;11(2):141-168. doi: $10.1038 / \mathrm{nrd} 3628$

20. Nierenberg AA, Husain MM, Trivedi MH, et al. Residual symptoms after remission of major depressive disorder with citalopram and risk of relapse: a STAR ${ }^{*}$ D report. Psychol Med. 2010;40(1):41-50. doi:10.1017/S0033291709006011

21. Davis MT, DellaGioia N, Matuskey D, et al. Preliminary evidence concerning the pattern and magnitude of cognitive dysfunction in major depressive disorder using cogstate measures. J Affect Disord. 2017;218:82-85. doi:10.1016/j.jad.2017.04.064

22. McIntyre RS, Best MW, Bowie CR, et al. The THINC-Integrated Tool (THINC-it) screening assessment for cognitive dysfunction: validation in patients with major depressive disorder. J Clin Psychiatry. 2017;78(7):873-881. doi:10.4088/JCP.16m11329 
23. Knight MJ, Baune BT. Cognitive dysfunction in major depressive disorder. Curr Opin Psychiatry. 2018;31(1):26-31. doi:10.1097/ YCO.0000000000000378

24. McIntyre RS, Lee Y. Cognition in major depressive disorder: a "Systemically Important Functional Index" (SIFI). Curr Opin Psychiatry. 2016;29(1):48-55. doi:10.1097/ YCO.0000000000000221

25. Russo M, Mahon K, Burdick KE. Measuring cognitive function in MDD: emerging assessment tools. Depress Anxiety. 2015;32 (4):262-269. doi:10.1002/da.22297

26. Wagner S, Doering B, Helmreich I, Lieb K, Tadić A. A meta-analysis of executive dysfunctions in unipolar major depressive disorder without psychotic symptoms and their changes during antidepressant treatment. Acta Psychiatr Scand. 2012;125(4):281292. doi:10.1111/j.1600-0447.2011.01762.x

27. Lee RSC, Hermens DF, Porter MA, Redoblado-Hodge MA. A metaanalysis of cognitive deficits in first-episode major depressive disorder. J Affect Disord. 2012;140(2):113-124. doi:10.1016/j. jad.2011.10.023

28. Lee RSC, Hermens DF, Naismith SL, et al. Neuropsychological and functional outcomes in recent-onset major depression, bipolar disorder and schizophrenia-spectrum disorders: a longitudinal cohort study. Transl Psychiatry. 2015;5:e555. doi:10.1038/ tp. 2015.50

29. Hasselbalch BJ, Knorr U, Hasselbalch SG, Gade A, Kessing LV. The cumulative load of depressive illness is associated with cognitive function in the remitted state of unipolar depressive disorder. Eur Psychiatry. 2013;28(6):349-355. doi:10.1016/j. eurpsy.2012.03.004

30. Hasselbalch BJ, Knorr U, Hasselbalch SG, Gade A, Kessing LV. Cognitive deficits in the remitted state of unipolar depressive disorder. Neuropsychology. 2012;26(5):642-651. doi:10.1037/ a0029301

31. Paelecke-Habermann Y, Pohl J, Leplow B. Attention and executive functions in remitted major depression patients. J Affect Disord. 2005;89(1-3):125-135. doi:10.1016/j.jad.2005.09.006

32. Miskowiak K, Vinberg M, Christensen EM, Kessing LV. Is there a difference in subjective experience of cognitive function in patients with unipolar disorder versus bipolar disorder? Nord J Psychiatry. 2012;66(6):389-395. doi:10.3109/08039488.2012.658862

33. Green MF. Cognitive impairment and functional outcome in schizophrenia and bipolar disorder. J Clin Psychiatry. 2006;67(10): e12.
34. Burdick KE, Goldberg JF, Harrow M. Neurocognitive dysfunction and psychosocial outcome in patients with bipolar I disorder at 15year follow-up. Acta Psychiatr Scand. 2010;122(6):499-506. doi:10.1111/j.1600-0447.2010.01590.x

35. Motter JN, Pimontel MA, Rindskopf D, Devanand DP, Doraiswamy PM, Sneed JR. Computerized cognitive training and functional recovery in major depressive disorder: a meta-analysis. J Affect Disord. 2016;189:184-191. doi:10.1016/j.jad.2015.09.022

36. Baune BT, Air T. Clinical, functional, and biological correlates of cognitive dimensions in major depressive disorder - rationale, design, and characteristics of the Cognitive Function and Mood Study (CoFaM-Study). Front Psychiatry. 2016;7:150. doi:10.3389/ fpsyt.2016.00150

37. Baune BT, Miller R, McAfoose J, Johnson M, Quirk F, Mitchell D. The role of cognitive impairment in general functioning in major depression. Psychiatry Res. 2010;176(2-3):183-189. doi:10.1016/j. psychres.2008.12.001

38. Clark M, DiBenedetti D, Perez V. Cognitive dysfunction and work productivity in major depressive disorder. Expert Rev Pharmacoecon Outcomes Res. 2016;16(4):455-463. doi:10.1080/14737167.2016.1195688

39. Cha DS, Carmona NE, Subramaniapillai M, et al. Cognitive impairment as measured by the THINC-integrated tool (THINC-it): association with psychosocial function in major depressive disorder J Affect Disord. 2017;222:14-20. doi:10.1016/j.jad.2017.06.036

40. Barlati S, Deste G, De Peri L, Ariu C, Vita A. Cognitive remediation in schizophrenia: current status and future perspectives. Schizophr Res Treat. 2013;2013:156084. doi:10.1155/2013/156084

41. Anguera JA, Gunning FM, Areán PA. Improving late life depression and cognitive control through the use of therapeutic video game technology: a proof-of-concept randomized trial. Depress Anxiety. 2017;34(6):508-517. doi:10.1002/da.22588

42. Arean PA, Hallgren KA, Jordan JT, et al. The use and effectiveness of mobile apps for depression: results from a fully remote clinical trial. J Med Internet Res. 2016;18(12):e330. doi:10.2196/jmir.6482

43. Oh SJ, Seo S, Lee JH, Song MJ, Shin M-S. Effects of smartphonebased memory training for older adults with subjective memory complaints: a randomized controlled trial. Aging Ment Health. 2018;22(4):526-534. doi:10.1080/13607863.2016.1274373

44. Howells A, Ivtzan I, Eiroa-Orosa FJ. Putting the 'app' in happiness: a randomised controlled trial of a smartphone-based mindfulness intervention to enhance wellbeing. Journal of Happiness Studies. 2016;17(1):163-185. doi:10.1007/s10902-014-9589-1 